\title{
Bilateral Sequential Pneumolabyrinth Resulting from Nose Blowing
}

\author{
Joong Seob Lee, Sae Young Kwon, Ji Heui Kim, and Hyung-Jong Kim \\ Department of Otorhinolaryngology-Head \& Neck Surgery, Hallym University Sacred Heart Hospital, Anyang, Korea
}

\begin{abstract}
Received September 8, 2015
Revised October 12, 2015

Accepted October 13, 2015
\end{abstract}

Pneumolabyrinth describes a condition with entrapped air in the labyrinth and usually occurs in temporal bone fractures that involve the otic capsule. While sporadic cases of bilateral pneumolabyrinth have been reported, cases lacking head trauma are very rare. We report the case of a 43-year-old man who had sudden hearing loss bilaterally after blowing his nose at an interval of 1 year. Although conservative management for the right ear and exploratory tympanotomy with sealing of the possible site of perilymphatic leakage in the left ear were performed, hearing outcome was poor in both ears. To our knowledge, this is the first case of bilateral pneumolabyrinth occurring as a result of nose blowing.

J Audiol Otol 2015;19(3):182-185

KEY WORDS: Pneumolabyrinth · Perilymphatic fistula · Hearing loss · Valsalva maneuver.

\section{Introduction}

Pneumolabyrinth manifests as air bubbles in the cochlear and/or vestibular labyrinth; its confirmative diagnosis is provided by high-resolution computed tomography (HRCT) of the temporal bone [1]. Although several causes of pneumolabyrinth have been reported, including temporal bone fracture, barotrauma, traumatic perilymphatic fistula (PLF), iatrogenic trauma resulting from ossiculoplasty, stapes surgery, and cochlear implant surgery [1-4], temporal bone fracture is regarded as the most common cause of pneumolabyrinth. While sporadic cases of bilateral pneumolabyrinth have been reported $[5,6]$, its bilateral occurrence without head trauma is rarely observed. We report a case of bilateral pneumolabyrinth caused by nasal blowing without any head trauma.

\section{Case Report}

A 43-year-old man visited the otolaryngology department

This is an Open Access article distributed under the terms of the Creative Commons Attribution Non-Commercial License (http://creativecommons. org/licenses/by-nc/3.0/) which permits unrestricted non-commercial use, distribution, and reproduction in any medium, provided the original work is properly cited. with complaints of ear fullness, hearing loss on the right side, and dizziness after blowing his nose, which began 1 day prior. He had a long history of rhinorrhea and nasal stuffiness. An otoscopic examination revealed that both tympanic membranes were intact. Pure tone audiometry showed 77-110 dB [bone-air hearing thresholds, speech discrimination (SD): $0 \%$ ] in his right ear and $20 \mathrm{~dB}$ (SD: 100\%) in the left ear.

HRCT of the temporal bone demonstrated right pneumolabyrinth, with air in both the semicircular canal and cochlea (Fig. 1A). In HRCT, the soft tissue densities were observed in both paranasal sinuses, as observed in chronic rhinosinusitis. In addition, soft tissue densities filled the enlarged Eustachian tubes bilaterally and extended to the tympanic orifice of each Eustachian tube (Fig. 1B).

Video nystagmography showed nonspecific nystagmus; however, apogeotropic nystagmus was observed during the head roll test, which was similar to a result of the left sided horizontal semicircular canal-benign paroxysmal positional vertigo. The patient was admitted to the hospital, and absolute bed rest was prescribed. He was also treated with prednisolone $(60 \mathrm{mg} /$ day) for 7 days. His vestibular symptoms resolved gradually, but his hearing showed no changes during his hospital stay. A follow-up HRCT of the temporal bones, 

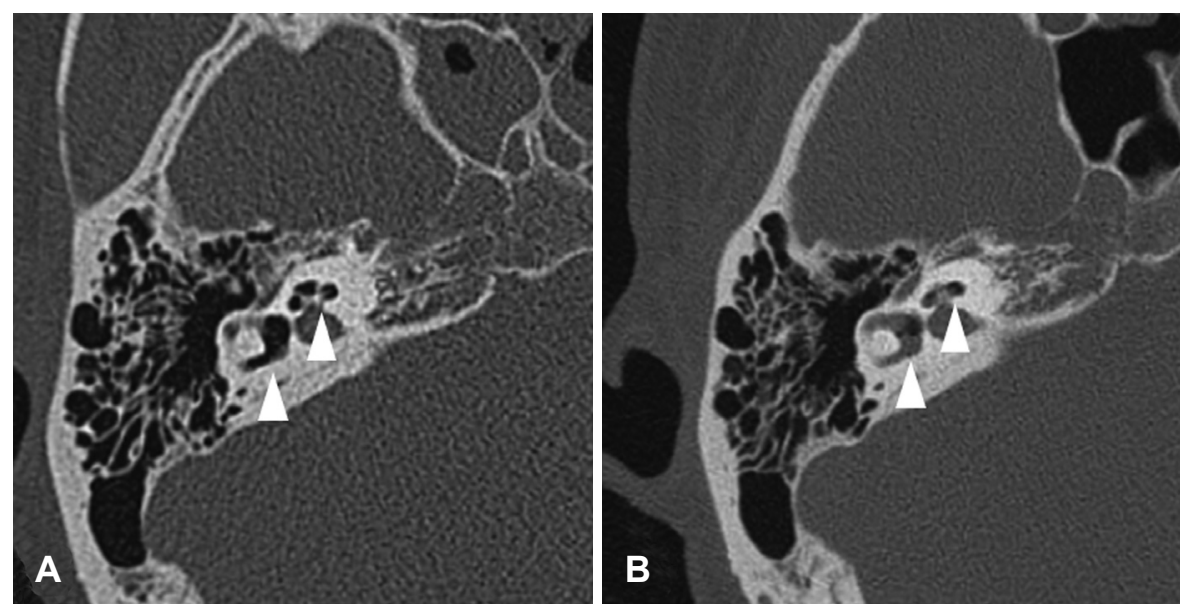

Fig. 1. CT images of the temporal bones. A: CT scan of the right temporal bone on the day of admission. Air bubbles (arrowheads) are observed in the cochlea and vestibule. B: CT scan of the right temporal bone on the sixth hospital day. Air bubbles (arrowheads) in the cochlea and vestibule are diminished. C: Axial CT scan of the temporal bones on the day of admission. Soft tissue densities (arrowheads) are visible in the both enlarged Eustachian tubes.
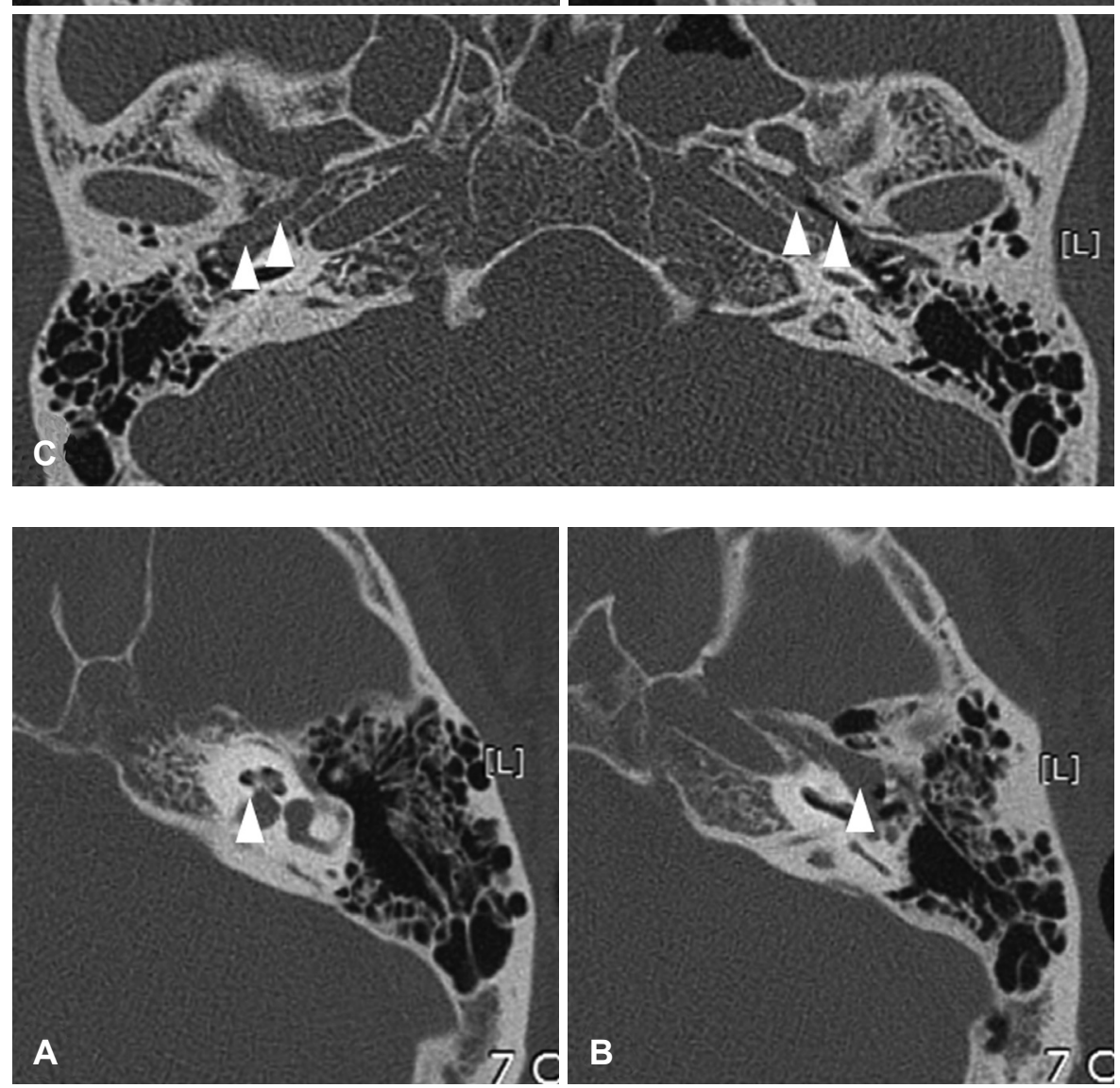

Fig. 2. CT images of the temporal bones at second admission. A: CT scan of the left temporal bone. Air bubbles (arrowhead) are visible in the cochlea. B: Soft tissue densities (arrowhead) are seen in the middle ear. C: Axial CT scan of the temporal bones. Enlarged Eustachian tubes are observed (arrowheads).

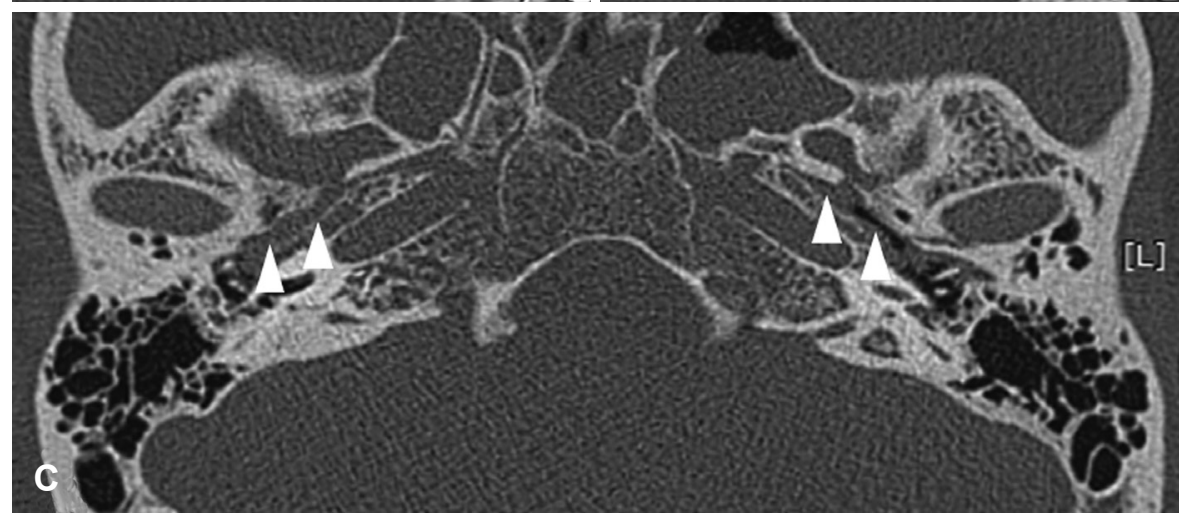


which was performed on the sixth hospital day, showed a substantial decrease in the number of air bubbles in the cochlea (Fig. 1C). The patient was discharged from the hospital with continuing prednisolone for 5 days.

One year later, the patient complained of ear fullness and hearing loss in his left ear (only hearing ear) after blowing his nose. However, he did not complain of any vertiginous sensations at that time. An otoscopic examination showed bilateral intact tympanic membranes. Pure tone audiometry demonstrated 77-98 dB (SD: 0\%) in the right ear, and 65-80 dB (SD: $36 \%$ ) in the left ear. HRCT of the temporal bones revealed air bubbles in the left cochlea (Fig. 2A), and soft tissue densities filled the Eustachian tubes bilaterally (Fig. 2B, C).

On the second hospital day, the patient received exploratory tympanotomy of the left ear with a suspected diagnosis of PLF. Surgery was performed under general anesthesia. During the surgery, both oval and round windows of the middle ear were observed to be covered by fibrous tissue, suspicious sites for PLF. Therefore, the two windows were tightly closed with tissue glue and soft tissues.

Pure tone audiometry demonstrated 43-48 dB (SD: 80\%) in the operated ear at postoperative day 8 . The patient was discharged from the hospital on that day.

One month after discharge, the patient complained of a progressive hearing disturbance in the left ear that persisted for 2 weeks. On otoscopic examination, the patient's tympanic membrane showed bilateral turbid colors. Pure tone audiometry showed a mixed-type hearing loss of the left ear (53$69 \mathrm{~dB}, \mathrm{SD}: 64 \%$ ). The deterioration of hearing was thought to be related to sensorineural hearing loss, which may be related to PLF and otitis media with effusion (OME). The deterioration was assumed to be the result of OME, because the patient had symptoms and HRCT findings of chronic rhinosinusitis. Therefore, the patient was treated with antibiotics and prednisolone.

Despite treatment, the patient demonstrated no significant improvement in his hearing. After 3 months, the patient agreed to wear a hearing aid in his left ear. He underwent myringotomy and ventilation tube insertion for otitis media. At present, 2 years after surgery, the patient wears his hearing aid well and regularly visits the otolaryngology clinic.

\section{Discussion}

PLF is highly suspected when a patient reports sudden hearing loss and dizziness with a history of otologic surgery or head trauma. There are two proposed routes for the development of PLF; explosive and implosive [7]. "Explosive" re- quires an increase in cerebrospinal fluid pressure. "Implosive" is caused by a Valsalva maneuver that produces an abrupt air pressure increase through the Eustachian tubes, a tear in the round window membrane, or the annular ligament of the stapes [7].

Yanagihara, et al. [8] reported a case of pneumolabyrinth induced by Eustachian tube air inflation. In that report, air likely entered the perilymphatic space through a preexisting fistula, forming static air pressure ranging from 165 to 545 $\mathrm{mm} \mathrm{H}_{2} \mathrm{O}$ which opened the Eustachian tube. The authors reported that either Politzer bag inflation or the Valsalva maneuver ranging between 250 to $650 \mathrm{~mm} \mathrm{H}_{2} \mathrm{O}$ could also cause a sufficient condition to push air into the inner ear and create pneumolabyrinth through a labyrinthine fistula. In the present case, PLF with pneumolabyrinth developed by barotrauma resulting from nose blowing, similar to the Valsalva maneuver. Although the exact mechanism remains unclear, we presume that the enlarged Eustachian tubes might play a role in the development of pneumolabyrinth in this case. The passage of air might be enhanced by the enlarged tubes, and airflow might be introduced into weak anatomical structures or a preexisting fistula. However, further evidence-based studies would be required to confirm this hypothesis.

There is no defined management of pneumolabyrinth due to the limited number of actual cases described [2]. Some physicians prefer conservative management, such as absolute bed rest, head elevation, and avoidance of the Valsalva maneuver $[9,10]$. However, if the patient shows aggravated vestibular symptoms and hearing loss, explorative tympanotomy is usually recommended $[9,10]$. In the present case, the vestibular symptoms of the patient improved quickly; therefore, we treated the patient with conservative management for the right ear. However, we performed explorative tympanotomy on the left ear. Because the affected ear was the only hearing ear at that time, we used an active management approach. Although the result of the surgery with this patient was not favorable, an early surgical approach might be helpful to patients in similar situations.

The reason for the worse hearing outcome is not clear. However, several factors may affect the hearing results in this patient. First, the poor bone conduction at disease onset would be associated with poor hearing outcome. Tsubota, et al. [11] proposed three predictive factors as bone conduction hearing level at disease onset, interval between injury and surgery, and presence of a stapes lesion, for hearing improvements in patients with pneumolabyrinth. In this patient, the bone conduction hearing level at the first visit was greater than $70 \mathrm{~dB}$. Thus, a poor hearing outcome was predicted. Second, the presence of air in the cochlea could be associated with a poor 
hearing outcome. A previous study [12] reported that air inside the cochlea could produce a severe reduction of cochlear potential and irreversible hearing loss. There is also a study [13] about air location and hearing outcomes in patients with traumatic pneumolabyrinth. In this report, among patients whose pneumolabyrinth was limited only to the vestibular organ, significantly larger numbers of patients recovered their hearing compared with patients with air inside the cochlea. Third, the recurrence of otitis media is associated with poor hearing outcome. Because the patient had chronic nasal disease, middle ear infection was an expected result.

There are several cases of bilateral pneumolabyrinth after head trauma $[14,15]$. However, there is no other report of bilateral pneumolabyrinth that occurred after nose blowing. Woo, et al. [2] reported that pneumolabyrinth without temporal bone fracture can be produced by an external force on the oval and round window membranes, fissula ante fenestram, microfissures, and Hyrtl's fissure. These were possible sites of PLF in this patient. However, further study of the anatomical and clinical differences is required to clarify the pathogenesis of this patient's bilateral pneumolabyrinth.

In conclusion, bilateral pneumolabyrinth can be produced by nasal blowing. A thorough history and diagnostic work up is mandatory, because there is a chance of irreversible hearing loss.

\section{REFERENCES}

1) Prisman E, Ramsden JD, Blaser S, Papsin B. Traumatic perilymphatic fistula with pneumolabyrinth: diagnosis and management. Laryngoscope 2011;121:856-9.
2) Woo HJ, Song SY, Kim YD, Bai CH. Pneumolabyrinth without temporal bone fracture: different outcomes for hearing recovery. Laryngoscope 2008;118:1464-6.

3) Pullen FW 2nd. Perilymphatic fistula induced by barotrauma. Am J Otol 1992;13:270-2.

4) Mun SK, Lee KH, Yang HS, Hong YH. Pneumolabyrinth resulting from trauma: report of two cases. Korean J Otorhinolaryngol-Head Neck Surg 2008;51:273-7.

5) Lee EJ, Yang YS, Yoon YJ. Case of bilateral pneumolabyrinth presenting as sudden, bilateral deafness, without temporal bone fracture, after a fall. J Laryngol Otol 2012;126:717-20.

6) Woodson EA, Smith RJ. Bilateral pneumolabyrinth diagnostic for otic capsule fractures without high-resolution imaging. Otolaryngol Head Neck Surg 2007;137:969-71.

7) Hornibrook J. Perilymph fistula: fifty years of controversy. ISRN Otolaryngol 2012;2012:281248.

8) Yanagihara N, Hyodo J, Takagi D, Miuchi S. A case of pneumolabyrinth induced by Eustachian tube air inflation. Otol Neurotol 2012; 33:1408-11

9) Yanagihara N, Nishioka I. Pneumolabyrinth in perilymphatic fistula: report of three cases. Am J Otol 1987;8:313-8.

10) Sarac S, Cengel S, Sennaroglu L. Pneumolabyrinth following traumatic luxation of the stapes into the vestibule. Int J Pediatr Otorhinolaryngol 2006;70:159-61.

11) Tsubota M, Shojaku H, Watanabe Y. Prognosis of inner ear function in pneumolabyrinth: case report and literature review. Am J Otolaryngol 2009;30:423-6.

12) Kobayashi $T$, Sakurada $T$, Ohyama $K$, Takasaka M. Inner ear injury caused by air intrusion to the scala vestibuli of the cochlea. Acta Otolaryngol 1993;113:725-30.

13) Hidaka H, Miyazaki M, Kawase T, Kobayashi T. Traumatic pneumolabyrinth: air location and hearing outcome. Otol Neurotol 2012; 33:123-31.

14) Lyos AT, Marsh MA, Jenkins HA, Coker NJ. Progressive hearing loss after transverse temporal bone fracture. Arch Otolaryngol Head Neck Surg 1995;121:795-9.

15) Woodson EA, Smith RJ. Bilateral pneumolabyrinth diagnostic for otic capsule fractures without high-resolution imaging. Otolaryngol Head Neck Surg 2007;137:969-71. 